\title{
ITERATIVELY DETECTED SPHERE PACKING MODULATED OFDM: AN EXIT CHART PERSPECTIVE
}

\author{
Lei Xu, Mohammed El-Hajjar, Osamah Alamri, Sheng Chen and Lajos Hanzo* \\ School of ECS, University of Southampton, SO17 1BJ, UK. \\ Email: $\{1 \mathrm{~h}\} @$ ecs.soton.ac.uk, http://www-mobile.ecs.soton.ac.uk
}

\begin{abstract}
A novel iteratively detected Sphere Packing (SP) modulation aided Orthogonal Frequency Division Multiplexing (OFDM) scheme is proposed, which we refer to as the SPOFDM arrangement. The advocated SP-OFDM system outperforms conventional OFDM dispensing with SP. As its upperbound performance limit, the Discrete Input Continuous Output Memoryless Channel (DCMC) capacity of this system is formulated. In order to maximize the DCMC channel capacity, a SP-to- OFDM-sub-carrier mapper (SPTSCM) is contrived, which is capable of providing an approximately $0.3 \mathrm{bit} / \mathrm{s} / \mathrm{Hz}$ DCMC capacity benefit, by employing a variety of different SPTSCM arrangements. Additionally, the proposed SP-OFDM scheme exhibits a DCMC capacity advantage of $0.8 \mathrm{bit} / \mathrm{s} / \mathrm{Hz}$ over the conventional equivalent-throughput Gray Mapping (GM) and Anti-Gray Mapping (AGM) based QPSK-OFDM schemes. Furthermore, the performance of the SP-OFDM system can be improved by serially concatenated convolutional coding relying on iterative extrinsic information exchange between the SP-symbol-to-bit demapper and the channel decoder. Explicitly, the proposed turbo-detected SP-OFDM scheme exhibits an approximately $E_{b} / N_{0}=3 \mathrm{~dB}$ and $E_{b} / N_{0}=4.5 \mathrm{~dB}$ gain at a Bit Error Ratio (BER) of $10^{-4}$ over the equivalentthroughput turbo-detected GM based QPSK-OFDM scheme and AGM QPSK-OFDM scheme, respectively. EXtrinsic Information Transfer (EXIT) charts are employed for analyzing the achievable convergence behaviour.
\end{abstract}

\section{INTRODUCTION}

In recent years, OFDM has found its way into a range of Wireless Local Area Network (WLAN) and Broadcast standards owing to its numerous benefits, such as for example that of converting frequency selective channels to parallel non-dispersive flat-fading channels. [1]

SP schemes [2] have the best-known minimum Euclidean distance in the multi-dimensional real-valued Euclidean space R [3], and hence they achieve a higher channel capacity, when the number of SP dimensions is increased. The concept of combining orthogonal transmit diversity designs with the principle of SP modulation was introduced by Su et al. in [3], where it was demonstrated that the proposed SP aided Space-Time Block-Coding (STBC) scheme was capable of outperforming the conventional orthogonal design based STBC schemes of [4].

The employment of the turbo principle was applied to the detection of spectrally efficient modulation schemes by several authors [5], where a soft symbol-to-bit demapper was used between the multilevel demodulator and the binary channel decoder. In [6], the turbo dectector employed in the SP aided STBC system was

${ }^{*}$ The financial support of the EPSRC, UK and the European Union under the auspices of the Newcom and Phoenix projects is gratefully acknowledged. demonstrated to be capable of further improving the attainable system performance with the aid of iterative detection at a cost of a commensurately increased complexity.

Motivated by these advances, we will propose a sophisticated scheme combining the freqency diversity of OFDM rather than the spatial diversity of STBC with SP modulation and derive the corresponding DCMC capacity equation. Furthermore, we exploit the benefits of iterative symbol-to-bit demapping and decoding. The soft SP-OFDM demapper was specifically designed for the sake of accepting the a priori information passed to it from the binary channel decoder as extrinsic information. As a benefit of the proposed solution, it will be demonstrated in Section 6 that the turbo detection aided SP-OFDM scheme is capable of providing an $E_{b} / N_{0}$ gain of $4.5 d B$ at a BER of $10^{-4}$. EXIT charts [7] are employed for analyzing the associated convergence behaviour and for calculating the channel capacity of the system.

This paper is organised as follows. In Section 2, a brief system overview is presented, followed by a description of the system design using SP-OFDm and its DCMC capacity equation in Section 3. The SP-symbol-to-bit demapper exploiting the a priori knowledge provided by the channel decoder is highlighted in Section 4. A brief introduction to EXIT charts is given in Section 5, while our simulation results and discussions are provided in Section 6. Finally, we conclude in Section 7.

\section{SYSTEM OVERVIEW}

The schematic of the entire system is shown in Fig.1, where the transmitted source bits are convolutionally encoded and then interleaved by a random bit interleaver. A rate $R=1 / 2$ NonSystematic Convolutional (NSC) code was employed. After channel interleaving, the SP modulator first maps $B$ number of channelcoded bits $\mathbf{b}=b_{0, \ldots, B-1} \in\{0,1\}$ to a SP symbol $\mathbf{s} \in \mathbf{S}$, so that we have $\mathbf{s}=\operatorname{map}_{s p}(\mathbf{b})$, where $B=\log _{2} L$. Subsequently, we have a set of SP symbols that are mapped to the OFDM subcarriers by an appropriately designed SPTSCM scheme. After SPTSCM mapping, the resultant sequence is serial-to-parallel converted and then modulated on to the $K$ OFDM sub-carriers by the classic Inverse Fast Fourier Transform (IFFT). Finally, the Cyclic Prefix (CP) is attached for protecting the OFDM signal from inter-symbol-interference (ISI) imposed by the multi-path channel. The complex Additive White Gaussian Noise (AWGN) of $n=n_{I}+j n_{Q}$ is also superimposed on the received signal, where $n_{I}$ and $n_{Q}$ are two independent zero mean Gaussian random variables having a variance of $\sigma_{n}^{2}=\sigma_{n_{I}}^{2}=\sigma_{n_{Q}}^{2}=N_{0} / 2$ per dimension, with $N_{0} / 2$ representing the double-sided noise power spectral density expressed in $W / H z$.

As shown in Fig.1, the received stream is first windowed to remove the CP and then OFDM demodulated by the FFT. Subsequently, the complex-valued OFDM sub-carrier symbols are demapped to SP symbols and then to their Log-Likelihood Ratio (LLR) representation for each of the $B$ coded bits per SP sym- 


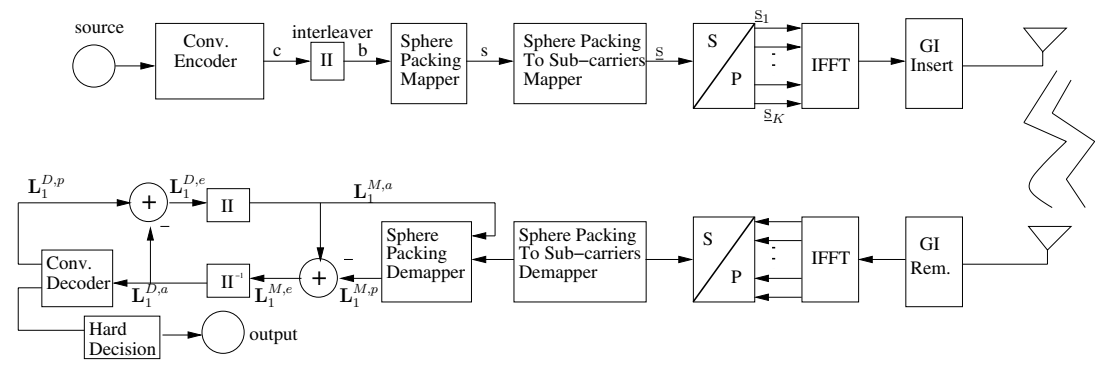

Figure 1: Schematic of the Turbo Detection Aided SP-OFDM System.

bol. The a priori LLR values $\mathbf{L}_{M, a}$ of the SP demodulator are subtracted from the a posteriori LLR values $\mathbf{L}_{M, p}$ for the sake of generating the extrinsic LLR values $\mathbf{L}_{M, e}$, and then the LLRs $\mathbf{L}_{M, e}$ are deinterleaved by a soft-bit deinterleaver, as seen in Fig.1. Next, the soft bits $\mathbf{L}_{D, a}$ are passed to the convolutional decoder in order to compute the a posteriori LLR values $\mathbf{L}_{D, p}$ provided by the Max-Log MAP algorithm [8] for all the channel-coded bits. During the last iteration, only the LLR values of the original uncoded systematic information bits are passed to a hard decision decoder in order to determine the estimated source bits. As usual, the extrinsic information $\mathbf{L}_{D, e}$, is generated by subtracting the $a$ priori information from the a posteriori information according to $\mathbf{L}_{D, p}-\mathbf{L}_{D, a}$, which is then fed back to the SP demapper as the a priori information $\mathbf{L}_{M, a}$ after appropriately reordering them using the interleaver of Fig.1. The SP demapper utilizes the a priori information for the sake of providing improved a posteriori LLR values, which are then passed to the channel decoder and then back to the demodulator for further iterations.

\section{OFDM SPHERE PACKING DESIGN}

In contrast to the STBC-SP design of [6], where the so-called diversity product quantifying the achievable coding advantage of an orthogonal transmit diversity scheme was maximised by employing SP modulation having the best known minimum Euclidean distance, here we exploit the SP scheme to improve the OFDM scheme's frequency diversity. The Frequency-Domain (FD) correlation between the OFDM subcarriers is a function of their FD separation. Hence it is necessary to investigate different SPTSCM schemes for attaining the best possible performance.

For the sake of generalising our treatment, let us assume that there are $L$ legitimate vectors $\left(x_{l, 1}, x_{l, 2}, \cdots, x_{l, M}\right), l=0,1$, $\ldots, L-1$, where $L$ represents the number of sphere-packed modulated symbols. The transmitter then has to choose the modulated signal from these $L$ legitimate symbols, which have to be transmitted over $M$ number of OFDM sub-carriers, where the throughput of the system is given by $\left(\log _{2} L\right) * K / M$ bits per OFDM symbol. In contrast to the conventional independent transmitted signals of the conventional individual OFDM sub-carriers, our aim is to design $x_{l, 1}, x_{l, 2}, \cdots, x_{l, M}$ jointly, so that they have the best minimum Euclidean distance from all other $(L-1)$ legitimate SP symbols, since this minimises the system's SP symbol error probability. Let $\left(a_{l, 1}, a_{l, 2}, a_{l, 3}, a_{l, 4}, \cdots, a_{l, 2 M-1}, a_{l, 2 M},\right), l=$ $0,1, \ldots, L-1$, be the legitimate phasor points of the $2 M$-dimensional real-valued Euclidean space $\mathrm{R}^{2 M}$. Hence, $\left(x_{l, 1}, x_{l, 2}, \cdots, x_{l, M}\right)$ may be written as

$$
\begin{aligned}
& \left\{x_{l, 1}, x_{l, 2}, \cdots, x_{l, M}\right\}=T\left(a_{l, 1}, a_{l, 2}, \cdots, a_{l, 2 M-1}, a_{l, 2 M},\right) \\
& =\left\{a_{l, 1}+j a_{l, 2}, a_{l, 3}+j a_{l, 4}, \cdots, a_{l, 2 M-1}+j a_{l, 2 M}\right\} .
\end{aligned}
$$

In the $2 \mathrm{M}$-dimensional real-valued Euclidean space $\mathrm{R}^{2 M}$, the lattice $D_{2 M}$ is defined as a sphere packing having the best minimum Euclidean distance from all other $(L-1)$ legitimate constellation points in $\mathrm{R}^{2 M}$ [2]. More specifically, $D_{2 M}$ may be defined as a lattice that consists of all legitimate sphere-packed constellation points having integer coordinates of $\left[\begin{array}{llllll}a_{1} & a_{2} & a_{3} & a_{4} & \ldots\end{array}\right.$ $\left.\begin{array}{lll}a_{2 M-1} & a_{2 M}\end{array}\right]$ uniquely and unambiguously describing the legitimate combinations of the modulated symbols $x_{l, 1}, \cdots, x_{l, M}$, but subjected to the sphere packing constraint of $a_{1}+a_{2}+\cdots+$ $a_{2 M-1}+a_{2 M}=O$, where $O$ is an even integer. Assuming that $\mathbf{S}$ $=\left\{\mathbf{s}^{l}=\left[a_{l, 1}, a_{l, 2}, \cdots, a_{l, 2 M-1}, a_{l, 2 M}\right] \in \mathrm{R}^{2 M}: 0 \leq l \leq L-1\right\}$ constitutes a set of $L$ legitimate constellation points belonging to the lattice $D_{2 M}$ having a total energy of $E \triangleq \sum_{l=0}^{L-1}\left(\left|a_{l, 1}\right|^{2}+\right.$ $\left.\left|a_{l, 2}\right|^{2}+\cdots+\left|a_{l, 2 M}\right|^{2}+\left|a_{l, 2 M}\right|^{2}\right)$, and upon introducing the notation

$$
C_{l}=\sqrt{\frac{M \cdot L}{E}}\left(x_{l, 1}, x_{l, 2}, \cdots, x_{l, M}\right), \quad l=0,1, \ldots, L-1,
$$

we have a set of $D_{2 M}$ SP constellation symbols, $\left\{C_{l}: 0 \leq l \leq\right.$ $L-1\}$, whose diversity product is determined by the minimum Euclidean distance of the set of $L$ legitimate constellation points in $\mathrm{S}$. The SP constellation symbols belonging to $D_{2 M}$ are transmitted with the aid of $M$ OFDM sub-carriers, thus the received vector corrupted by the frequency-selective Rayleigh fading channel and the AWGN can be represented as:

$$
\begin{gathered}
\mathbf{y}=\left[y^{1}, y^{2}, \cdots, y^{M}\right]^{T}=\sqrt{\frac{M \cdot L}{E}} \mathbf{h} \cdot \mathbf{s}^{l}+n=\operatorname{diag}\left[h_{1}, h_{2}, \cdots,\right. \\
\left.h_{M}\right] \cdot\left[x_{l, 1}, x_{l, 2}, \cdots, x_{1, M}\right]^{T}+\left[n_{1}, n_{2}, \cdots, n_{M}\right]^{T},
\end{gathered}
$$

where $\mathbf{y}$ is the complex-valued received signal vector, constituted by the signals $y^{1}, y^{2}, \cdots, y^{M}$ at the output of the $M$ employed OFDM sub-carriers, while $h_{1}, h_{2}, \cdots, h_{M}$ are their stationary and complex-Gaussian distributed Frequency Domain CHannel Transfer Factors (FDCHTF) having a zero-mean and a unit variance. Furthermore, $n_{1}, n_{2}, \cdots, n_{M}$ are zero-mean complex-valued Gaussian random variables having variances of $\sigma_{1}^{2}=\sigma_{2}^{2}=\cdots=$ $\sigma_{M}^{2}=N_{0}$.

The conditional probability of receiving an $M$-dimensional complex-valued signal vector $\mathbf{y}$, given the transmitted SP symbol of $\mathbf{s}^{l}$ and the FDCHTF $\mathbf{h}$ is determined by:

$$
P\left(\mathbf{y} \mid \mathbf{s}^{l}, \mathbf{h}\right)=\frac{1}{\left(\pi N_{0}\right)^{M}} \exp \left(-\frac{\left\|\mathbf{y}-\alpha \mathbf{s}^{l}\right\|^{2}}{N_{0}}\right),
$$

where we have $\alpha=\mathbf{h} \cdot \sqrt{M \cdot L / E}$.

The channel capacity associated with SP signalling, when transmitting the SP symbols of the lattice $D_{M}$ over the Discrete-Input Continuous-Output Memoryless Channel (DCMC) can be derived from that of the Discrete Memoryless Channel (DMC) as:

$$
\begin{aligned}
C_{D C M C}^{S P}= & \max _{p\left(\mathbf{s}^{1}\right) \cdots p\left(\mathbf{s}^{L}\right)} \sum_{l=1}^{L} \underbrace{\int_{-\infty}^{+\infty} \cdots \int_{-\infty}^{+\infty}}_{2 M-\text { fold }} p\left(\mathbf{y} \mid \mathbf{h}, \mathbf{s}^{l}\right) p\left(\mathbf{s}^{l}\right) p(\mathbf{h}) \\
& \log _{2}\left(\frac{p\left(\mathbf{y} \mid \mathbf{h}, \mathbf{s}^{l}\right)}{\sum_{n=1}^{L} p\left(\mathbf{y} \mid \mathbf{h}, \mathbf{s}^{n}\right) p\left(\mathbf{s}^{n}\right)}\right) d \mathbf{y} d \mathbf{h}
\end{aligned}
$$

where we exploited that the FDCHTF $\mathbf{h}$ is independent of $\mathbf{s}^{l}, l \in$ $[1, \cdots, L-1]$. Considering the correlations between the $M$ specific sub-carriers conveying a SP symbol, the general Probability Density Function (PDF) $p(\mathbf{h})$ of an $M$-dimensional complexGaussian variable $\mathbf{h}$ can be expressed as:

$$
p(\mathbf{h})=\frac{1}{(2 \pi)^{M} \operatorname{det}(\Sigma)} \exp \left[-\left(\mathbf{h}-\mathbf{m}_{\mathbf{h}}\right)^{H} \Sigma^{-1}\left(\mathbf{h}-\mathbf{m}_{\mathbf{h}}\right) / 2\right]
$$


where $\mathbf{m}_{\mathbf{h}}=E[\mathbf{h}]$ is the first-order mean of the complex-Gaussian random vector $\mathbf{h}, \Sigma=(1 / 2) E\left[\left(\mathbf{h}-\mathbf{m}_{\mathbf{h}}\right)\left(\mathbf{h}-\mathbf{m}_{\mathbf{h}}\right)^{H}\right]$ is its nonsingular covariance matrix having $(M \times M)$ elements, $\operatorname{det}(\Sigma)$ is the determinant of $\Sigma$, and $\mathbf{h}^{H}$ represents the Hermitian transpose of $\mathbf{h}$. Since $\mathbf{h}$ has a zero-mean, the $i$-th row and $j$-th column element of $\Sigma$ may be written as:

$$
\Sigma_{i, j}=(1 / 2) E\left(h_{i} h_{j}^{*}\right),
$$

where $h_{j}^{*}$ is the conjugate of $h_{j}$. The correlation between $h_{i}$ and $h_{j}^{*}$ is given by [9]:

$$
E\left\{h_{i} h_{j}^{*}\right\}=\int_{-\infty}^{+\infty} E\left\{h(\tau) h^{*}(\tau)\right\} e^{-j 2 \pi \Delta f} d \tau,
$$

where $\tau$ represents the time-lag and $\Delta f$ is the FD seperation between the specific OFDM sub-carriers $\left(h_{i}, h_{j}\right)$ within the same OFDM symbol. The correlation function is a monotonically decreasing function of the FD seperation between the sub-carriers. When the FD seperation is significantly higher than the coherence bandwith, the correlation is considered to be zero. Let us now substitute Eq.(8) into Eq.(7) and use both Eq.(5) and Eq.(6), which allows us to conclude that the right hand side of Eq.(5) is maximised, when $p\left(\mathbf{s}^{l}\right)$ represents the uniform distribution and the FDCHTF's correlation is minimized, provided that no channel information is available at the transmitter. By contrast, if the FDCHTF's correlation is known at the transmitter, this may be exploited for optimizing the SPTSCM component. Therefore, we should allocate the specific sub-carriers conveying a particular SP symbol as far apart from each other as possible, when designing the SPTSCM scheme. A rectangular interleaver can be utilized for creating a regular mapping scheme for assigning the same fixed sub-carrier spacing to the $M$ selected sub-carriers conveying the same SP symbol. Our simulation results associated with different values of the sub-carrier spacing will confirm this expectation, as demonstrated in Section6.

\section{ITERATIVE DEMAPPING}

In order to provide extrinsic information for the Soft-In Soft-Out (SISO) channel decoder for the sake of attaining an iteration gain, the SP demapper has to transform the soft information from the probabilities of the legitimate SP symbols to those of the corresponding bits. According to Eq.(3), the received SP symbol $\mathbf{y}$ carries $B$ channel-coded bits $\mathbf{b}=b_{0, \ldots, B-1} \in\{0,1\}$. If perfect FDCHTF knowledge is assumed to be available at the receiver, the LLR-value of bit $k$ for $k=0, \ldots, B-1$ can be written as [10]

$$
L\left(b_{k} / \mathbf{y}\right)=L_{a}\left(b_{k}\right)+\ln \frac{\sum_{\mathbf{s}^{l} \in S_{1}^{k}} p\left(\mathbf{y} / \mathbf{s}^{l}, \mathbf{h}\right) \cdot e^{\sum_{j=0, j \neq k}^{B-1} b_{j} L_{a}\left(b_{j}\right)}}{\sum_{\mathbf{s}^{l} \in S_{0}^{k}} p\left(\mathbf{y} / \mathbf{s}^{l}, \mathbf{h}\right) \cdot e^{\sum_{j=0, j \neq k}^{B-1} b_{j} L_{a}\left(b_{j}\right)}},
$$

where $\mathbf{S}_{1}^{k}$ and $\mathbf{S}_{0}^{k}$ are subsets of the SP symbol constellation $\mathbf{S}$, so that $\mathbf{S}_{1}^{k} \triangleq\left\{\mathbf{s}^{l} \in \mathbf{S}: b_{k}=1\right\}$ and likewise, $\mathbf{S}_{0}^{k} \triangleq\left\{\mathbf{s}^{l} \in \mathbf{S}\right.$ : $\left.b_{k}=0\right\}$. In other words, $\mathbf{S}_{i}^{k}$ represents all SP symbols of the set $\mathbf{S}$, where we have $b_{k} \in\{0,1\}, k=0, \ldots, B-1$. Using Eq.(4), we can write Eq.(9) as

$$
\begin{aligned}
& L\left(b_{k} / \mathbf{y}\right)=L_{a}\left(b_{k}\right) \\
& +\ln \frac{\sum_{\mathbf{s}^{l} \in \mathbf{S}_{1}^{k}} \exp \left[-\frac{1}{N_{0}}\left\|\mathbf{y}-\alpha \cdot \mathbf{s}^{l}\right\|^{2}+\sum_{j=0, j \neq k}^{B-1} b_{j} L_{a}\left(b_{j}\right)\right]}{\sum_{\mathbf{s}^{l} \in \mathbf{S}_{0}^{k}} \exp \left[-\frac{1}{N_{0}}\left\|\mathbf{y}-\alpha \cdot \mathbf{s}^{l}\right\|^{2}+\sum_{j=0, j \neq k}^{B-1} b_{j} L_{a}\left(b_{j}\right)\right]} \\
& =L_{M, a}+L_{M, e} \text {. }
\end{aligned}
$$

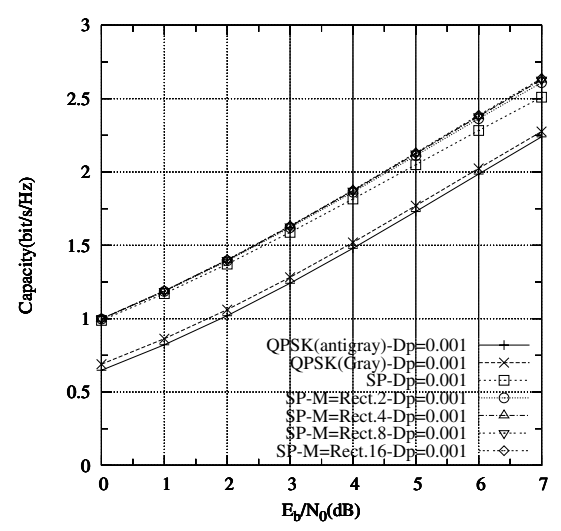

Figure 2: DCMC Channel Capacity Comparison of the QPSK-OFDM and SP-OFDM Schemes Using the System Parameters Outlined in Tab.1.

Finally, the max-log approximation of Eq.(10) is as follows

$$
\begin{aligned}
& L\left(b_{k} / \mathbf{y}\right)=L_{a}\left(b_{k}\right) \\
& +\max _{\mathbf{s}^{l} \in \mathbf{S}_{1}^{k}}\left[-\frac{1}{N_{0}}\left\|\mathbf{y}-\alpha \cdot \mathbf{s}^{l}\right\|^{2}+\sum_{j=0, j \neq k}^{B-1} b_{j} L_{a}\left(b_{j}\right)\right] \\
& -\max _{\mathbf{s}^{l} \in \mathbf{S}_{0}^{k}}\left[-\frac{1}{N_{0}}\left\|\mathbf{y}-\alpha \cdot \mathbf{s}^{l}\right\|^{2}+\sum_{j=0, j \neq k}^{B-1} b_{j} L_{a}\left(b_{j}\right)\right] .
\end{aligned}
$$

Thus we have derived the soft a posteriori information $L_{M, p}$ as shown in Fig.1, which will then be passed to the channel decoder after subtracting $L_{M, a}$ from it.

\section{EXIT CHART ANALYSIS}

EXIT charts were proposed by ten Brink [7] for analyzing the convergence characteristics of turbo codes as a convenient visualization technique. This technique computes the mutual information of the output extrinsic and input a-priori components corresponding to the associated bits $s$ for each of the iterative SISO blocks. More specifically, the mutual information of the LLRs and the legitimate bipolar BPSK bits is given by [7]:

$\mathbf{I}=\frac{1}{2} \sum_{c \in\{+1,-1\}} \int_{-\infty}^{\infty} p_{L}(x \mid c) \cdot \log _{2}\left[\frac{2 p_{L}(x \mid c)}{p_{L}(x \mid+1)+p_{L}(x \mid-1)}\right] d x$,

where $p_{L}(x \mid c)$ is the conditional probability distribution of the LLRs. We can now substitute the conditional probability distribution of the extrinsic information $L_{e}$ and that of the a-priori information $L_{a}$ into Eq.(12) in order to derive the mutual information between either $\mathbf{I}_{e}$ or $\mathbf{I}_{a}$ and the legitimate bipolar bits, respectively. The required distribution of the LLRs in Eq.(12) can be approximated by the typically more accurate experimentally generated LLR histogram or by using the potentially less computationally demanding but possibly less accurate Gaussian distribution for modeling the LLR distribution.

In this contribution, the latter technique is employed for analyzing the convergence behaviour of our system. Despite the potentially non-Gaussian LLR distribution, the EXIT chart analysis will be shown to be sufficiently accurate.

\section{RESULTS AND DISCUSSION}

Without loss of generality, we considered a SP modulation scheme associated with $L=16$ SP symbols and using $M=2$ OFDM subcarriers in order to demonstrate the performance improvements achieved by the proposed system. All the system parameters used in the simulations are listed in Tab.1. 


\begin{tabular}{|l|r|}
\hline Modulation & Sphere Packing with $L=16$ \\
\hline Dimension of Sphere Packing & $2 M=4$ \\
\hline Channel & Correlated Rayleigh Fading \\
\hline $\begin{array}{l}\text { Normalised Doppler } \\
\text { frequency }\end{array}$ & $f_{D}=0.001$ \\
\hline Outer channel Code & NSC, $(2,1,3)$ \\
\hline Interleaver Length & 1310720 \\
\hline Number of subcarriers of OFDM & 64 \\
\hline Length of GI & 16 \\
\hline System throughput & 2 bit/sub-carrier \\
\hline
\end{tabular}

Table 1: System parameters

Since the multi-dimensional signal of Eq.(2) is multiplied by a factor that is inversely proportional to $\sqrt{E}$, namely by $\sqrt{M \cdot L / E}$, it is desirable to choose that specific subset of $L=16 \mathrm{SP}$ points from the entire set of legitimate SP constellation points hosted by $D_{4}$, which results in the minimum total energy. It was shown in [2] that there is a total of 24 legitimate SP symbols ${ }^{1}$ hosted by $D_{4}$ having an identical minimum energy of $E=2$. We used a computer search for determining the optimum choice of the $L=16$ SP points out of the possible 24 points, which possess the highest minimum Euclidean distance, hence minimising the SP symbol error probability.

Fig.2 provides a DCMC capacity comparison between conventional QPSK modulation and the proposed SP-OFDM scheme, which was plotted for Eq.5. The results are presented for the $D_{4}$ SP constellation of $L=16$ and using the system parameters of Tab.1. The mutual information was calculated for Eq.12 and then integrated using the histogram-based method to obtain the simulation based channel capacity. It is seen in Fig. 2 that the DCMC capacities of both GM and AGM QPSK modulation are the same, although their iterative performances are different. The proposed SP-OFDM scheme is capable of achieving an approximately 0.7 bit/s/Hz channel capacity gain over that of GM or AGM aided QPSK modulation. When the FD seperation between the two OFDM sub-carriers conveying a SP symbol is increased, a higher channel capacity can be attained. However, the upper bound of the channel capacity associated with the lowest possible correlation between the two sub-carriers conveying a specific SP symbol is closely approached, when the spacing between the sub-carriers is higher than four sub-carriers. This confirms our previous analysis carried out using Eq.5. It is also notable that the channel capacity gain attained by reducing the correlation between the two sub-carriers of a SP symbol becomes more pronounced, when the SNR value is high. This is due to the fact that frequency diversity succeeds in counteracting the FD fading, but unable to mitigate the effects of interference and AWGN when all the received signals are heavily contaminated.

Fig.3 illustrates the associated EXIT characteristics for both the QPSK modulation scheme and the SP-OFDM arrangement in conjunction with different SPTSCM regimes at $E_{b} / N_{0}=7 d B$. As expected, GM does not provide any iteration gain upon increasing the mutual information at the input of the demapper, while the AGM provides substantial iteration gains, when provided with improved a priori information. The various different SP and AGM QPSK schemes characterized in Fig.3 have a similar level of start-

\footnotetext{
${ }^{1}$ In simple terms, the sphere centred at $(0,0,0,0)$ has 23 spheres around it, centred at the points $(+/-1,+/-1,0,0)$, where any choice of signs and any ordering of the coordinates is legitimate [ [2], p.9].
}

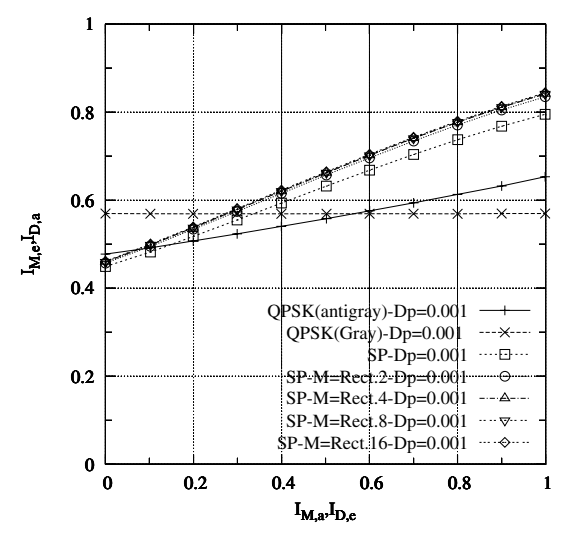

Figure 3: EXIT curves of both the GM and AGM based conventional QPSK-OFDM scheme compared to an identical-throughput SP-OFDM scheme with $L=16$ at $E_{b} / N_{0}=7 d B$. The remaining system parameters were outlined in Tab.1.

ing point at $I_{M, a}=0$, but they exhibit different slopes upon increasing the a priori information. Observe in Fig.3 that the SPOFDM schemes outperform the conventional OFDM approach. Again, as expected, the SP scheme having a larger spacing between the two OFDM subcarriers of a SP symbol demonstrates a higher potentional iteration gain.

Fig.4 gives an illustration of the actual decoding trajectory of the turbo-detected NSC-encoded SP-OFDM scheme using a subcarrier spacing of four for transmission over a correlated Rayleigh channel having an OFDM-symbol-normalized Doppler frequency of $f_{D}=0.001$ at $E_{b} / N_{0}=7 d B$. The zigzag-path marked with arrows indicates the extrinsic information exchange between the SP demapper and the outer NSC decoder. The iterative detection process commences from the origin of Fig.4, which represents the absence of a priori information for the SP demapper. Then the decoding trajectory traverses to the demapper's EXIT curve in Fig.4, indicating that valuable LLR information was generated by the demapper. The resultant extrinsic information is forwarded to the channel decoders and hence the detection trajectory reaches the EXIT curve of the channel decoder, which demonstrates that further extrinsic information is obtained from the SISO channel decoder assisted by the a priori information gleaned from the demapper. During its further evolution, the trajectory traces up to the demapper's EXIT curve again, as a result of using the extrinsic information generated by the channel decoder, which is then fed back to the demapper and so forth. In other words, the trajectory evolves in this manner within the open detection tunnel between the EXIT curves of the demapper and the channel decoder, until it reaches the intersection of the curves. Since the simulated detection trajectories basically traverse back and forth between the EXIT curves of the receiver components by closely matching it, the validity of EXIT chart analysis is demonstrated.

Fig.5 compares the attainable BER performance of the proposed SP OFDM scheme equipped with different SPTSCM components and that of a conventional identical-throughput 2 Bit Per sub-carrier (2BPS) QPSK-OFDM design using both AGM and GM of the coded bits, when communicating over a correlated Rayleigh fading channel having an OFDM-symbol-normalized Doppler frequency of $f_{D}=0.001$ and an interleaver length of $\mathbf{D}=1,310,720$ bits. Observe in Fig.5 that the GM based QPSK-OFDM BER curves are similar, regardless of the number of iterations, as predicted by its flat EXIT curve seen in Fig.3. By contrast, AGM aided QPSK-OFDM achieved a substantial BER performance im- 
provement in conjunction with iterative demapping and decoding, although the BER performance recorded for AGM after the first iteration is worse than that of the GM. Explicitly, a coding advantage of about $1.5 d B$ was achieved at a BER of $10^{-4}$ after $I=7$ iterations by the AGM aided QPSK-OFDM system over the GM based QPSK-OFDM scheme. Additionally, a further significant coding advantage was attained over the AGM QPSK-OFDM approach, when employing SP. Specifically, an approximately $3 d B$ coding advantage was realized at a BER of $10^{-4}$ after $I=7$ iterations. It is also observed in Fig.5 that the FD spacing between the two sub-carriers conveying the same SP symbols affects the achievable system performance exactly in the same manner as expected. Explicitly, the lower the correlation between the pair of sub-carrier FDCHTFs, the better the attainable performance of the system.

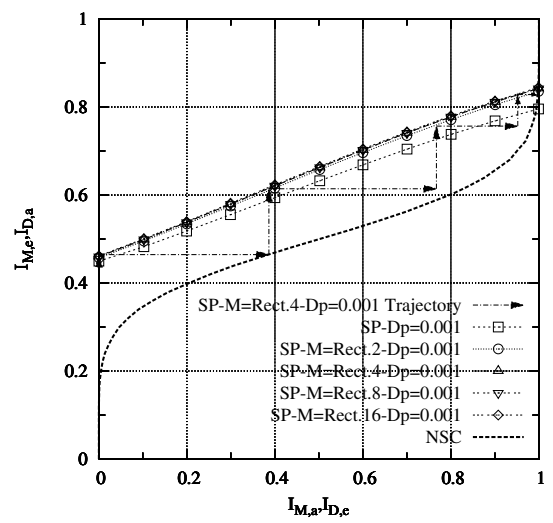

Figure 4: Decoding trajectory of the turbo-detected NSC coded SPOFDM scheme employing an $L=16$-ary SP symbols conveyed by $M=2$ sub-carriers having a sub-carriers spacing of four ad employing the system parameters listed in Tab.1.

\section{CONCLUSION}

In this paper we proposed a novel system that exploits the advantages of both iterative demapping and turbo detection [10], as well as those of the SP-OFDM scheme developed. The proposed SP-OFDM scheme benefits from a substantial frequency diversity gain, provided that the $L=16$-ary SP symbols are conveyed by subcarriers having uncorrelated FDCHTFs. This was ensured by a FD spacing of four in the scenario considered. The

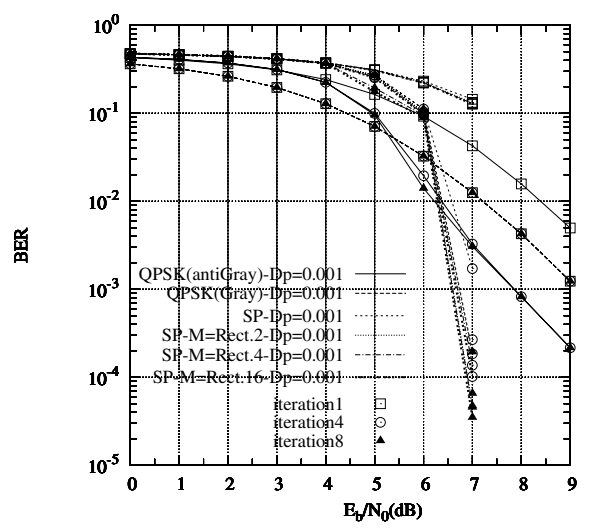

Figure 5: BER performance comparison of the GM and AGM based convolutional-coded QPSK-OFDM schemes against an identicalthroughput 2BPS coded SP-OFDM scheme invoking $I t=7$ iterations and using the system parameters outlined in Tab.1. theoretic DCMC capacity equation of this system was provided for assisting in the SPTSCM design. Furthermore, our investigations demonstrated that significant performance improvements may be achieved, when the SP-OFDM scheme is combined with an outer channel codec and iterative SP-symbol-to-bit demapping, when compared to the conventional QPSK-OFDM based benchmark system. When using an appropriate SPTSCM scheme and $I=7$ turbo detection iterations, an $E_{b} / N_{0}$ gain of about $4.5 \mathrm{~dB}$ and a capacity enhancement of $0.8 b i t / s / H z$ were attained by the SP-OFDM scheme over the identical-throughput QPSK-OFDM benchmarker scheme.

\section{REFERENCES}

[1] L. Hanzo, M. Münster, B. J. Choi and T. Keller, OFDM and $M C$ CDMA. West Sussex, England: John Wiley and IEEE Press, 2003.

[2] J. H. Conway and N. J. Sloane, Sphere Packings, Lattices and Groups. Springer-Verlag, 1999.

[3] W. Su, Z. Safar, and K. J. R. Liu, "Space-time signal design for timecorrelated Rayleigh fading channels," in IEEE International Conference on Communications, vol. 5, (Anchorage, Alaska), pp. 31753179, 2003.

[4] S. Alamouti, "A simple transmit diversity technique for wireless communications," IEEE Journal on Selected Areas in Communications, vol. 16, no. 8, pp. 1451-1458, 1998.

[5] L. Hanzo, T. H. Liew, and B. L. Yeap, Turbo Coding, Turbo Equalisation and Space-Time Coding: for Transmission over Fading Channels. Chichester, England: John Wiley and Sons Ltd and IEEE Press, NY, USA, 2002.

[6] O. Alamri, B. J. YEAP, and L. Hanzo, "A turbo detection and spherepacking-modulation-aided space-time coding scheme," IEEE Transactions on Vehicular Technology, vol. 2007, no. 56, pp. 575-582, 2007.

[7] S. ten Brink, "Convergence of iterative decoding," IEE Electronics Letters, vol. 35, no. 13, pp. 1117-1118, June 1999.

[8] P. Robertson, E. Villebrun, and P. Hoeher, "A comparison of optimal and sub-optimal MAP decoding algorithms operating in the log domain," in Proceedings of International Conference on Communications, (Seattle, USA), pp. 1009-1013, Jun 1995.

[9] Bultitude and R.J.C, "Estimating frequency correlation functions from propagation measurements on fading radio channels: a critical review," IEEE Journal On Selected Areas in Communications, vol. 20, pp. 1133-1143, Aug. 2002.

[10] S. ten Brink, J. Speidel, and R.-H. Yan, "Iterative demappping and decoding for multilevel modulation," in IEEE Global Telecommunications Conference, vol. 1, (Sydney, Australia), pp. 579-584, 8-12 Nov 1998. 